\begin{tabular}{ll} 
Abstract EPV090/\#629 Table 1 & Patient details \\
\hline Parameters & Total(\%) \\
\hline $\mathrm{n}(\%)$ & $111(100 \%)$ \\
Age in years & 57.52 \\
Stage 1 2A 2B 3A 3B 4A 4B & $30(16 \%) 1(0.05 \%) 44(25 \%) 1(0.05 \%)$ \\
& $22(12 \%) 1(0.05 \%) 13(7 \%)$ \\
Chemo-radiation Radiation(RT) Surgery Surgery - & $58(52 \%) 11(9 \%) 11(9 \%) 12(10 \%) 1$ \\
Adjuvant NACT * - surgery NACT ${ }^{*}-\mathrm{RT}$ & $(0.9 \%) 1(0.9 \%) 2(1.8 \%) 9(8 \%)$ \\
Chemotherapy Palliative treatment & \\
\hline
\end{tabular}

demographics, treatment modalities and outcomes were analyzed in 111 patients who had treatment in this institution.

Results The mean age of the patients was 57.52 years (Range 20-84 years). Demographics and treatment details are presented in the attached table (table 1).The median follow up period was 20 months. The median overall survival in stage 1 , stage 2, stage 3 and stage 4 are 37(IQR:17-60), 28(IQR: $12.5-40.5), 11.5(\mathrm{IQR}: 7-34)$ and 9 (IQR:5-16) months respectively. The median survival for the entire cohort was 22 months (IQR : 9-48).

Conclusions This present study shows poorer survival when compared to squamous cell carcinoma in literature. The benefit of addition of chemotherapy or surgery as salvage to the present standard of care needs to be studied prospectively in a larger population.

\section{EPV091/\#647 TREATMENT OF LOCAL AND LOCO-REGIONAL RECURRENCES IN LOCALLY ADVANCED CERVICAL CANCER, RETROSPECTIVE STUDY}

'D Cantu-De Leon, ${ }^{2} \mathrm{~L}$ Gallardo-Alvarado, ${ }^{1} \mathrm{G}$ Moreno*, ${ }^{2} \mathrm{~J}$ Galicia. ${ }^{1}$ Instituto Nacional de Cancerologia, Gynecology Oncology, Mexico, Mexico; ${ }^{2}$ Instituto Nacional de Cancerologia, Clinical Research, Mexico, Mexico

\subsection{6/ijgc-2021-IGCS.159}

Objectives Recurrent disease in the central pelvis following radiation therapy may potentially be cured with pelvic excenteration (PE) procedure. However, there are other options of treatment according to the characteristics of the patients. The aim of this study is to describe treatments and clinical outcomes in patients with isolated pelvic failures after definitive radiation treatment for cervicalcancer.

Methods Cervical cancer patients with isolated pelvic failure after definitive radiation with brachytherapy (RT) were identified in a tertiary academic center from 2005 to 2014. Isolated failures in the cervix or pelvic nodes were biopsy-proven, and had a compute tomography without distant metastasis.

Results Isolated pelvic failure was detected in $79(7.6)$ out of 1046 consecutive patients treated RT. The median time to isolated pelvic recurrence was 15 months (range 3-153). Median follow-up time for patients alive after isolated pelvic recurrence was 49 months (range 2-181). of these 79 patients, 19 (24.1\%) have PE has elective treatment but only 3 (3.8\%) received this procedure, $3(3.8 \%)$ patients had radical hysterectomy ( 2 patients by original treatment plan and one did not accept PV), 32 (40.5\%) was candidate for SC and receive this treatment. 3 patients $(3.8 \%)$ was candidate palliative care but 13 (16.5\%) receive chemotherapy. 24 patients (30.4\%) did not receive other treatment, or rejected treatment. Median OS for patients treated with surgery, chemotherapy, or palliative care or not was 20 months (14-145), 9 months (2-12), respectively.

Conclusions Locoregional recurrence could be cured by pelvic excenteration, but most of the patient did not accept the treatment compromising the overall survival.

\section{EPV092/\#74 TODAY'S PREVENTABLE CANCERS: HPV VACCINATION KNOWLEDGE AND UPTAKE IN HEALTH PROFESSIONAL GRADUATE STUDENTS (HPS)}

${ }^{1} \mathrm{M}$ Levy ${ }^{*},{ }^{2} \mathrm{P}$ Novoa-Vogt, ${ }^{2} \mathrm{M}$ Castillo, ${ }^{2} \mathrm{M}$ Huang. ${ }^{1}$ University of Miami Miller School of Medicine, Department of Medical Education, Miami, USA; ${ }^{2}$ Sylvester Comprehensive Cancer Center, Department of Gynecologic Oncology, Miami, USA

\subsection{6/ijgc-2021-IGCS. 160}

Objectives HPV vaccination is safe and effective for cancer prevention, but uptake remains low. Very little data exists on attitudes and knowledge of HPV vaccines among HPS. Edification of HPS knowledge will improve their ability to educate patients.

Methods This cross-sectional survey study of HPS (medical, public health, nursing) assessed knowledge of HPV, HPV vaccine, and vaccine uptake. The study was IRB approved (\#20201459). Data analysis was conducted in SPSS.

Results 234 students completed the survey with more students identifying as female (Table). Knowledge of the HPV vaccine differed significantly between professions $(\mathrm{p}<0.001)$ and was significantly higher in medicine compared to both nursing ( $\mathrm{p}$ $<0.001)$ and public health $(\mathrm{p}<0.01)$. Knowledge of HPV differed significantly between professions $(\mathrm{p}<0.001)$ and was significantly higher in medicine compared to both nursing ( $\mathrm{p}$ $<0.001)$ and public health $(\mathrm{p}<0.005)$. There were no knowledge differences between the nursing and public health schools. Nursing and public health students largely were unaware that the latest age to receive vaccination is $44.92 \%$ of participants initiated the HPV vaccine series, but only $61 \%$ completed the vaccine series.

Abstraact EPV092/\#74 Table 1 Demographics, vaccination status, and knowledge by school

\begin{tabular}{|c|c|c|c|}
\hline & $\begin{array}{c}\text { Medicine } \\
\mathrm{n}=141(18 \%)\end{array}$ & $\begin{array}{c}\text { Nursing } \\
\mathrm{n}=54(14 \%)\end{array}$ & $\begin{array}{l}\text { Public Health } \\
\mathrm{n}=39(35 \%)\end{array}$ \\
\hline $\begin{array}{l}\text { Biological Sex } \\
\text { Female } \\
\text { Male }\end{array}$ & $\begin{array}{l}108(77) \\
33(23)\end{array}$ & $\begin{array}{c}48(89) \\
6(11)\end{array}$ & $\begin{array}{c}35(90) \\
4(10)\end{array}$ \\
\hline $\begin{array}{l}\text { HPV Related Cancer Risk Factors } \\
\text { Used Oral Contraceptive Pills } \\
\text { Have Smoked } \\
\text { Sexually active }\end{array}$ & $\begin{array}{c}85(60) \\
1(1) \\
120(85)\end{array}$ & $\begin{array}{c}33(61) \\
2(4) \\
41(76)\end{array}$ & $\begin{array}{l}27(69) \\
2(5) \\
31(80)\end{array}$ \\
\hline HPV vaccine knowledge, median (SD) & $0.800(0.188)$ & $0.634(0.211)$ & $0.634(0.199)$ \\
\hline Initiated HPV vaccine Series & $112(79)$ & $38(70)$ & $33(85)$ \\
\hline Completed HPV vaccine Series & $93(66)$ & $24(46)$ & $27(69)$ \\
\hline HPV knowledge, median (SD) & $0.846(0.104)$ & $0.769(0.122)$ & $0.769(0.106)$ \\
\hline $\begin{array}{l}\text { Condoms are effective in preventing } \\
\text { against HPV. (False) }\end{array}$ & $29(21)$ & $16(30)$ & $7(18)$ \\
\hline $\begin{array}{l}\text { HPV is spread through blood or other } \\
\text { bodily fluid. (False) }\end{array}$ & $61(43)$ & $12(22)$ & $16(41)$ \\
\hline $\begin{array}{l}\text { Oldest age for HPV vaccine } \\
15 \\
26 \\
34 \\
44 \text { (correct) }\end{array}$ & $\begin{array}{c}8(6) \\
27(19) \\
15(11) \\
86(61)\end{array}$ & $\begin{array}{c}4(7) \\
19(35) \\
5(9) \\
24(44)\end{array}$ & $\begin{array}{c}5(13) \\
16(41) \\
4(10) \\
13(3)\end{array}$ \\
\hline
\end{tabular}


Conclusions HPS across schools demonstrated gaps in knowledge surrounding both HPV and the HPV vaccine. Specifically students knew HPV causes cervical cancer; however, major knowledge gaps persist in the prevention of HPV infection. These areas represent high-yield opportunities for improvement within HPS education to ensure dissemination of knowledge regarding cancer prevention.

\section{EPV093/\#95 CLINICAL CHARACTERISTICS, TREATMENT RESPONSE AND PROGNOSIS OF LOCALLY ADVANCED ADENOCARCINOMA OF THE CERVIX, A LOCAL STUDY}

M Yu*. Jose R. Reyes Memorial Medical Center, Obstetrics and Gynecology, Manila, Philippines

\subsection{6/ijgc-2021-IGCS. 161}

Objectives The objective of this study is to determine the clinical characteristics, treatment response and prognosis of locally advanced adenocarcinoma of the cervix who underwent concurrent chemoradiotherapy with weekly Cisplatin or Carboplatin in comparison to squamous cell carcinoma.

Methods Outpatient charts of the cervical cancer patients from the outpatient department of Section of Gynecologic Oncology of a tertiary hospital were retrospectively reviewed.

Results Among the 979 charts reviewed, only 278 patients were included in the analysis. Seventy-five percent of the patients had squamous cell carcinoma and only 20\% had adenocarcinoma. Baseline characteristics were comparable. Ninety-eight percent had Cisplatin-based concurrent chemoradiotherapy. Median follow up was 17 months, with $75 \%$ of the patients had complete response and $16 \%$ had recurrent disease. Most common site of recurrence was cervix, lungs and bones. Disease free survival and overall survival was the same for adenocarcinoma and squamous cell carcinoma.

Conclusions Patients with locally advanced adenocarcinoma of the cervix who underwent concurrent chemoradiation had the same treatment response and prognosis to patients with squamous cell carcinoma.

\section{EPV093a/\#176 A MACHINE LEARNING APPROACH APPLIED TO GYNECOLOGICAL ULTRASOUND TO PREDICT PROGRESSION-FREE SURVIVAL IN OVARIAN CANCER PATIENTS}

${ }^{1}$ F Arezzo, ${ }^{2} \mathrm{~V}$ Loizzi, ${ }^{3} \mathrm{G}$ Cazzato, ${ }^{1} \mathrm{C}$ Lombardi, ${ }^{4} \mathrm{~V}$ Venerito, ${ }^{1} \mathrm{E}$ Cicinelli, ${ }^{1} \mathrm{G}$ Cormio ${ }^{*}$ ${ }^{1} \mathrm{CM}$ Santarsiero. ${ }^{1}$ Policlinico of Bary, Obstetrics and Gynecology Department, Bari, Italy; 2University of Bari 'Aldo Moro', Interdisciplinar Department of Medicine, Bari, Italy; ${ }^{3}$ Department of Emergency and Organ Transplantation, Pathology Section, Bari, Italy; ${ }^{4}$ Department of Emergency and Organ Transplantation, Rheumatology Unit, Bari, Italy

\subsection{6/ijgc-2021-IGCS.162}

Objectives Ultrasound(US) is a cheap, non-invasive and wellrecognized image modality for diagnosing and assessing ovarian cancer(OC). However, approximately $18 \%$ to $31 \%$ of adnexal lesions detected on US remain indeterminate. Machine learning(ML) is a promising tool for the implementation of complex multi-parametric algorithms. Despite the standardization of features capable of supporting the discrimination of ovarian masses into benign and malignant, there is the lack of accurate predictive modeling based on US examination for progression-free survival (PFS).

Methods In this retrospective observational study, we analyzed patients with epithelial ovarian cancer(EOC) who were followed in a tertiary center from 2018 to 2019. Demographic, clinical and laboratory characteristics were collected as well as information about post-surgery histopathology. Furthermore, we recorded data about US examinations according to International Ovarian Tumor Analysis(IOTA) classification. Proper feature selection was used to determine an attribute core set. Random Forest(RFF) algorithm was trained and validated with 10 -fold cross-validation to predict 12-month PFS. The accuracy of the algorithm was than assessed scoring accuracy and Area Under Receiver Operating Characteristic(AUROC).

Results Our analysis included n.32OC patients with mean age of $54.1 \pm 14.9$ years at diagnosis. Histotypes were n.19/32 (59.4\%) serous carcinoma, n.5/32(15.6\%) mucinous, n.5/32 (15.6\%) endometriod and n.3/32(9.4\%) clear cell. All patients underwent radical surgery. The attribute core set used to train machine learning algorithms is reported in figure 1. RFF showed an accuracy of 0.81 , AUROC 0.91 .

Conclusions We developed an accurate model to predict 12month PFS in patients with OC based on a ML algorithm applied to gynecological ultrasound evaluation, requiring few easy-to-collect attributes.

\section{EPV093b/\#769 PARADIGM SHIFT TO SENTINEL LYMPH NODE BIOPSY IN ENDOMETRIAL CANCER SURGERY: RECENT U.S. TRENDS}

${ }^{1}$ A Vallejo*, ${ }^{2} \mathrm{M}$ Klar, ${ }^{2} \mathrm{M}$ Hasanov, ${ }^{1} \mathrm{~K}$ Ciesielski, ${ }^{1} \mathrm{~L}$ Muderspach, ${ }^{1,3} \mathrm{~L}$ Roman, ${ }^{4} \mathrm{~J}$ Wright,

${ }^{1,3} \mathrm{~K}$ Matsuo. 'University of Southern California, Division Gynecology Oncology, Department of Obstetrics and Gynecology, Los Angeles, USA; ${ }^{2}$ University of Feibrug Faculty of Medicine, Obstetrics and Gynecology, Frieburg, Germany; ${ }^{3}$ University of Southern California, Norris Comprehensive Cancer Center, Los Angeles, USA; ${ }^{4}$ Columbia University College of PHysicians and Surgeons, Gynecologic Oncology, New York, USA

\subsection{6/ijgc-2021-IGCS. 163}

Objectives This study examined the population-level uptakes and outcomes of sentinel lymph node (SLN) biopsy for early endometrial cancer.

Methods This retrospective observational study queried the Surveillance, Epidemiology, and End Results Program, examining 83,139 women with endometrial cancer who underwent primary hysterectomy with lymph node evaluation for T1 disease from 2003-2018. Main outcome measures were (i) temporal trends and patient characteristics in utilization of SLN biopsy, and (ii) endometrial cancer-specific mortality associated with SLN biopsy.

Results The utilization of SLN biopsy increased significantly from $0.2 \%$ to $29.7 \%$ from $2005-2018$ with robust increase after $2016(\mathrm{P}<0.001$; top-panel). The uptake of SLN biopsy was higher for endometriod histology $(0.3 \%$ to $31.6 \%$ between 2005-2018) versus non-endometriod histology $(0.6 \%$ to $21.0 \%$ between $2006-2018$ )(both, $\mathrm{P}<0.001$ ). In a multivariable analysis, recent year surgery, endometrioid histology, well-differentiated tumors, T1a disease, and smaller tumor size remained independent characteristics for SLN biopsy use (all, $\mathrm{P}<0.05$ ). Performance of SLN biopsy was not associated with increased endometrial cancer-specific mortality compared to lymphadenectomy in endometrioid hystology (subdistribution- 